\title{
Long-term outcome in early survivors of cardiogenic shock at the acute stage of myocardial infarction: a landmark analysis from the French registry of Acute ST-elevation and non-ST-elevation Myocardial Infarction (FAST-MI) Registry
}

\author{
Nadia Aissaoui ${ }^{1,2}$, Etienne Puymirat ${ }^{1,2,3}$, Tabassome Simon $^{4,5,6}$, Eric Bonnefoy-Cudraz ${ }^{7}$, Denis Angoulvant ${ }^{8}$, \\ Francois Schiele ${ }^{9}$, Hakim Benamer ${ }^{10}$, Philippe Quandalle ${ }^{11}$, Fabrice Prunier ${ }^{12}$, Eric Durand ${ }^{13}$, Laurence Berard ${ }^{4}$, \\ Didier Blanchard ${ }^{14}$ and Nicolas Danchin ${ }^{1,2^{*}}$
}

\begin{abstract}
Introduction: There are little data about patients with cardiogenic shock (CS) who survive the early phase of acute myocardial infarction (AMI). The aim of this study was to assess long-term (5-year) mortality among early survivors of AMI, according to the presence of CS at the acute stage.

Methods: We analyzed 5-year follow-up data from the French registry of Acute ST-elevation and non-ST-elevation Myocardial Infarction (FAST-MI) 2005 registry, a nationwide French survey including consecutive patients admitted for ST or non-ST-elevation AMI at the end of 2005 in 223 institutions.

Results: Of 3670 patients enrolled, shock occurred in 224 (6.1\%), and 3411 survived beyond 30 days or hospital discharge, including 99 (2.9\%) with shock. Early survivors with CS had a more severe clinical profile, more frequent concomitant in-hospital complications, and were less often managed invasively than those without CS. Five-year survival was 59\% in patients with, versus 76\% in those without shock (adjusted hazard ratio $(H R)=1.72$ [1.24-2.38], $P=0.001)$. The excess of death associated with CS, however, was observed only during the first year (one-year survival: 77\% vs 93\%, adjusted HR: 2.87 [1.85 to 4.46] $P<0.001$ ), while survival from one to 5 years was similar (76\% vs 82\%, adjusted HR: 1.06 [0.64 to 1.74]). Propensity score-matched analyses yielded similar results.
\end{abstract}

Conclusions: In patients surviving the early phase of AMI, CS at the initial stage carries an increased risk of death up to one year after the acute event. Beyond one year, however, mortality is similar to that of patients without shock.

Trial registration: ClinicalTrials.gov number, NCT00673036, Registered May 5, 2008.

\section{Introduction}

Despite considerable progress in the management of patients admitted for acute myocardial infarction (AMI), cardiogenic shock (CS) remains a major complication $[1,2]$. In-hospital mortality has declined due to early revascularization and improved overall management, however,

\footnotetext{
* Correspondence: nicoals.danchin@egp.aphp.fr

'Department of Cardiology, Assistance Publique-Hôpitaux de Paris, Hôpital Européen Georges Pompidou, Paris, France

${ }^{2}$ Faculty of Medicine, University Paris Descartes, Paris, France

Full list of author information is available at the end of the article
}

early mortality rates remain high; only about $50 \%$ of AMI patients developing CS are alive at one month [1-4].

Little data exist on the long-term outcomes of patients surviving the acute phase of CS following AMI [4-6]. Most studies report that patients with CS have an increased risk of death up to one year after the acute event. Beyond 1 year, mortality seems to be similar to that of patients without CS. However, most of these studies were performed in the 1990s., Marked reductions in AMI mortality have recently been reported, including among patients with CS [4-6]. 
The French registry of Acute ST-elevation and nonST-elevation Myocardial Infarction (FAST MI) 2005 registry is a prospective, nationwide, observational study conducted at the end of 2005 in a large number of the French hospitals treating AMI patients [7,8]. It allows evaluation of the long-term outcome in acute ST-elevation and non-ST-elevation myocardial infarction patients with and without CS. The aim of the present study was to analyse long-term outcomes of early survivors of the acute phase, according to the presence of CS at the acute stage.

\section{Materials and methods \\ Study population}

The methods of the FAST-MI 2005 registry have been described in detail elsewhere $[7,8]$. Briefly, the primary objectives were to evaluate myocardial infarction (MI) management in real-life practice and to assess short- and long-term outcomes of patients admitted to ICUs for MI. Patients were consecutively recruited from ICU departments over a period of 1 month (October to November 2005), with a 1-month extension for patients with known diabetes mellitus. Participation in the study was offered to all French institutions, university teaching hospitals, general and regional hospitals, and private clinics with ICUs authorized to receive acute coronary syndromes (ACS). In each centre, a physician was in charge of the registry and provided a full list of all patients admitted to the unit. The number of participating centres was 223, representing $60 \%$ of all centres taking care of AMI patients in France at that time.

Inclusion criteria were (1) men or women, over 18 years old; (2) patients admitted within $48 \mathrm{~h}$ after symptom onset in an ICU for an AMI characterized by increased troponin or creatine kinase-MB associated with at least one of the following elements: symptoms compatible with myocardial ischaemia, appearance of pathologic Q-waves, or ST-T changes compatible with myocardial ischaemia (ST-segment elevation or depression, T-wave inversion); and (3) consent to take part in the study. Patients who died very soon after admission and for whom cardiac markers were not measured were included if they had compatible signs or symptoms associated with typical ST-segment changes. Exclusion criteria were (1) refusal to participate; (2) patients with MI who were admitted more than $48 \mathrm{~h}$ after symptom onset; (3) patients with iatrogenic MI, defined as MI occurring within $48 \mathrm{~h}$ of a therapeutic procedure (bypass surgery, coronary angioplasty, or any other medical or surgical intervention); (4) ACS diagnosis invalidated in favour of another diagnosis; and (5) patients with unstable angina and no increase in cardiac biomarkers.

CS was defined as systolic blood pressure $<90 \mathrm{~mm} \mathrm{Hg}$ for $\geq 1 \mathrm{~h}$ not responsive to fluid administration alone, thought to be secondary to cardiac dysfunction, and associated with signs of hypoperfusion or cardiac index $\leq 2.2 \mathrm{l} / \mathrm{min} / \mathrm{mm} 2$ and pulmonary capillary wedge pressure $>18 \mathrm{~mm} \mathrm{Hg}[9,10]$.

All patients provided informed consent for their participation in the registry. The protocol was reviewed by the Committee for the Protection of Human Subjects in Biomedical Research of St Antoine University Hospital and the data file of the study was declared to and authorized by the French data protection committee (Commission Nationale Informatique et Liberté). Participating physicians were asked not change their usual therapeutic approach for the purpose of the survey. All the authors vouch for the fidelity of the study to the trial protocol, which is available at ClinicalTrials.gov number, NCT00673036 (Registered 5 May 2008).

Overall, 3,670 patients were included in the survey. Among them, 224 (6.1\%) developed shock: 259 patients died in hospital or during the first 30 days following admission, and 3,411 patients survived the early phase (patients surviving the initial hospital stay and alive at 30 days) and were included in the present study.

\section{Data collection}

Baseline characteristics, namely demographics (age, gender), risk factors (hypertension, body mass index $>30 \mathrm{~kg} /$ $\mathrm{m}^{2}$, diabetes, current smoking, hyperlipidaemia, family history), medical history (previous AMI, previous percutaneous coronary intervention (PCI), previous coronary artery bypass grafting, previous stroke, previous heart failure, prior peripheral arterial disease, previous chronic renal failure, previous chronic obstructive pulmonary disease and previous cancer), and previous medications (antiplatelet agents, statins, angiotensin-converting enzyme (ACE) inhibitors, angiotensin-receptor blockers (ARB)s, beta-blockers and insulin) were collected prospectively and stored electronically as previously described.

Clinical presentation, and glycaemia at the time of admission were also collected and the last value of left ventricular ejection fraction (LVEF) during the hospital stay was recorded. We also recorded the use of cardiac procedures, in-hospital complications (re-infarction, stroke, major bleeding, the need for transfusion, ventricular fibrillation, new atrial fibrillation and second-and third-degree atrio-ventricular (AV) block) and medications (antiplatelet agents, diuretics, beta-blockers, ACE-inhibitors and lipidlowering agents) used in the first $48 \mathrm{~h}$ and at hospital discharge in early CS survivors.

\section{Outcome}

Mortality was assessed at 1 and 5 years both in patients with and without CS, who were discharged alive and were alive at one month (early survivors). Follow up was centralised at the French Society of Cardiology and dedicated research technicians contacted both physicians and patients 
themselves, after checking patients' vital status in municipal registries. Causes of death were obtained by direct contact with patients' physicians or families, and from the national causes of death registry. Two cardiologists, unaware of patients' hospital course, adjudicated causes of death as cardiovascular, non-cardiovascular, or unknown. The rate of patients lost to follow up was $0.3 \%$ at 1 year, $2 \%$ at 3 years and $5 \%$ at 5 years.

\section{Statistical analysis}

Statistical analysis was performed using IBM SPSS 20.0 (IBM SPSS, Inc., Armonk, NY, USA) and NCSS 9.0.7 (NCSS, LLC. Kaysville, UT, USA). For quantitative variables, mean and standard deviations were calculated. Discrete variables are presented as percentages. Comparisons were performed with the chi-square or Fisher's exact test for discrete variables and with the unpaired $t$-test, or Wilcoxon sign-rank tests for continuous variables. Odds ratios (OR) or hazard ratios (HR) are presented with the 95\% CI. Five-year mortality rates were calculated using the Kaplan-Meier method and comparisons were made using log-rank tests.

Because coronary artery disease remains unstable for several months after an acute coronary syndrome, and potent antithrombotic medications, such as dual antiplatelet therapy, are recommended for 1 year following AMI, we selected the 1-year time point for performing landmark analyses: the analyses were performed with 1 -year survival as the dependent variable in the population of early survivors, and 5-year survival as the dependent variable in the population of patients alive at one year [11].

Correlates of 5-year mortality were determined using a multivariate Cox backward analysis. The cumulative hazard functions for each covariable were computed to assess proportionality, and colinearity was verified by calculating variance inflation factors. Shock was analysed as a timedependent variable, with the time-point set at 12 months from the acute episode. Variables included in the final multivariate models were selected ad hoc, based upon their physiological relevance and potential to be associated with outcomes; thus, we included variables likely to influence outcome negatively (age, history of heart failure, history of diabetes, history of prior AMI, history of stroke, history of peripheral artery disease, anemia on admission) or positively (history of hypertension, current smoking, revascularisation by percutaneous coronary intervention or surgery, early use of low molecular weight heparin, glycoprotein IIB-IIIa inhibitors, and discharge medications: aspirin, clopidogrel, statins, ACE-inhibitors, ARBs, beta-blockers) as well as sex, type and region of institution, and type of MI (segment-elevation myocardial infarction (STEMI) versus non-segment-elevation myocardial infarction (NSTEMI).
In addition, to assess the potential role of CS on late mortality, we calculated propensity scores for having presented CS, using logistic regression analysis 1) in patients alive at hospital discharge and at 30 days, and 2) in patients who were alive at one year $(c$-statistic $=0.76$ for both propensity scores). Cohorts with and without CS were constituted, matched on the propensity scores (3-to-1 matching: population of early survivors, 94 patients with CS, 282 patients without CS; population of 1-year survivors, 73 patients with CS, 217 patients without CS), and their outcomes were compared using logrank tests. For calculating the propensity scores, we used baseline characteristics, early management including revascularisation procedures and antithrombotic medications, in-hospital complications (re-infarction and major bleeding) and discharge medications. LVEF and medications indicated for heart failure (renin angiotensin aldosterone inhibitors and beta-blockers) were not included in the model to avoid over-adjustment. For all analyses, a $P$-value $<0.05$ was considered significant.

\section{Results}

\section{Baseline characteristics of all 30-day survivors}

Among the 3,411 early survivors, 99 (2.9\%) had developed CS at the acute stage. Patients with CS were significantly older and had more comorbid conditions (Table 1). Clinical presentation at admission of patients with CS was more severe, and they had developed more complications during hospitalization. The use of revascularization procedures was comparable in the two groups.

\section{Five-year survival}

Five-year survival was $59 \%$ in early survivors with CS, compared with $76 \%$ in early survivors without shock $(P<0.001)$ (Figure 1).

\section{Landmark analyses in the overall population}

One-year survival was $93 \%$ in early survivors without CS and $77 \%$ in patients with CS. Cause of death analysis showed that cardiovascular death was as frequent in CS patients $(65.2 \%)$ as in non-CS patients (66.3\%). Five-year survival was $76 \%$ in the 76 CS patients surviving at one year, versus $82 \%$ in the 3,072 non-CS patients surviving at one year (an additional table shows this in more detail; see Additional file 1). Beyond 1 year, there was a non-significant trend towards increased cardiovascular mortality in CS patients (55.6\% versus $36.3 \%$ ), but with fewer deaths of unknown cause (22.2\% versus $31.1 \%$ ).

In the time-dependent Cox multivariate model, CS was independently associated with increased hazard for death at 12 months (HR: 2.87, 95\% CI: 1.85, 4.46, $P<0.001$ ), but carried no increased risk from 1 year to 5 years (HR 1.06, 95\% CI: 0.64, 1.74). 
Table 1 Baseline characteristics of patients surviving at $\mathbf{3 0}$ days and hospital discharge according to presence of cardiogenic shock at the acute stage

\begin{tabular}{|c|c|c|c|}
\hline & No shock $(n=3,312)$ & Shock $(n=99)$ & $P$-value \\
\hline Age, years, mean $\pm S D$ & $66 \pm 14$ & $70 \pm 13$ & $<0.001$ \\
\hline Sex, female, n (\%) & $1014(30.6)$ & $37(37.4)$ & 0.43 \\
\hline Body mass index, $\mathrm{Kg} / \mathrm{m}^{2}$, mean $\pm \mathrm{SD}$ & $27.2 \pm 4.7$ & $26.5 \pm 4.9$ & 0.15 \\
\hline \multicolumn{4}{|l|}{ Risk factors, n (\%) } \\
\hline Hypertension & $1949(58.8)$ & $62(62.6)$ & 0.45 \\
\hline Diabetes mellitus & $1166(35.2)$ & $37(37.4)$ & 0.65 \\
\hline Current smoking & $990(29.9)$ & $27(27.3)$ & 0.59 \\
\hline Hypercholesterolemia & $1622(49.0)$ & $44(44.4)$ & 0.37 \\
\hline Family history of coronary artery disease & $796(24.0)$ & $11(11.1)$ & 0.003 \\
\hline \multicolumn{4}{|l|}{ Previous medical history, n (\%) } \\
\hline Myocardial infarction & $591(17.8)$ & $23(23.2)$ & 0.17 \\
\hline Percutaneous coronary intervention & $472(14.3)$ & $13(13.1)$ & 0.75 \\
\hline Coronary artery bypass graft & $186(5.6)$ & $8(8.1)$ & 0.30 \\
\hline Stroke & $168(5.1)$ & $5(5.1)$ & 0.99 \\
\hline Peripheral arterial disease & $316(9.6)$ & $17(17.2)$ & 0.01 \\
\hline Heart failure & $172(5.2)$ & $9(9.1)$ & 0.09 \\
\hline Chronic kidney disease & $172(5.2)$ & $6(6.1)$ & 0.71 \\
\hline Chronic obstructive pulmonary disease & $141(4.3)$ & $9(9.1)$ & 0.02 \\
\hline Cancer & $218(6.6)$ & $4(4.0)$ & 0.31 \\
\hline \multicolumn{4}{|l|}{ Previous medications, n (\%) } \\
\hline Antiplatelet agents & $1058(31.9)$ & $35(35.4)$ & 0.47 \\
\hline Statins & $930(28.1)$ & $32(32.4)$ & 0.35 \\
\hline Angiotensin-converting enzyme inhibitor & $640(19.3)$ & $33(33.3)$ & 0.001 \\
\hline Angiotensin receptor blockers & $515(15.5)$ & $13(13.1)$ & 0.52 \\
\hline Beta-blockers & $828(25.0)$ & $28(28.3)$ & 0.46 \\
\hline Insulin & $335(10.1)$ & $13(13.1)$ & 0.33 \\
\hline \multicolumn{4}{|l|}{ Current episode, n (\%) } \\
\hline \multirow[t]{2}{*}{ Typical chest pain } & $2538(79.0)$ & $58(65.2)$ & 0.002 \\
\hline & $(n=3213)$ & $(n=89)$ & \\
\hline Resuscitated cardiac arrest & $31(0.9)$ & $10(10.1)$ & $<0.001$ \\
\hline Segment-elevation myocardial infarction & $1688(50.9)$ & $54(54.5)$ & 0.47 \\
\hline Anaemia on admission & $689(21.5)(n=3202)$ & $35(36.8)(n=95)$ & $<0.001$ \\
\hline Admission glycaemia, mg/dl, mean \pm SD & $156 \pm 77$ & $203 \pm 109$ & $<0.001$ \\
\hline Left ventricular ejection fraction, $\%$, mean \pm SD & $53 \pm 13$ & $42 \pm 16$ & $<0.001$ \\
\hline \multicolumn{4}{|l|}{ Medications within the first $48 \mathrm{~h}, \mathrm{n}(\%)$} \\
\hline Low molecular-weight heparin & $2159(65.1)$ & $45(45.5)$ & $<0.001$ \\
\hline Clopidogrel & $2882(86.9)$ & $81(81.8)$ & 0.14 \\
\hline GP IIb-IIla inhibitors & $1236(37.3)$ & $35(35.4)$ & 0.69 \\
\hline \multicolumn{4}{|l|}{ Procedures during hospital stay, n (\%) } \\
\hline Coronary angiography & $2898(87.4)$ & 79 (79.8) & 0.03 \\
\hline Percutaneous coronary intervention & $2186(65.9)$ & $60(60.9)$ & 0.27 \\
\hline Coronary artery bypass graft & $140(4.2)$ & $4(4.0)$ & 0.93 \\
\hline
\end{tabular}


Table 1 Baseline characteristics of patients surviving at $\mathbf{3 0}$ days and hospital discharge according to presence of cardiogenic shock at the acute stage (Continued)

\begin{tabular}{|c|c|c|c|}
\hline \multicolumn{4}{|l|}{ In-hospital complications, n (\%) } \\
\hline Re-infarction & $51(1.5)$ & $3(3.0)$ & 0.24 \\
\hline Stroke & $19(0.6)$ & $4(4.0)$ & $<0.001$ \\
\hline Major bleeding & $54(1.6)$ & $7(7.1)$ & $<0.001$ \\
\hline Transfusion & $119(3.6)$ & $13(13.1)$ & $<0.001$ \\
\hline Ventricular fibrillation & $44(1.3)$ & $12(12.1)$ & $<0.001$ \\
\hline Atrial fibrillation, new & $150(4.5)$ & $25(25.3)$ & $<0.001$ \\
\hline Atrio-ventricular block & $39(1.2)$ & $4(4.0)$ & 0.01 \\
\hline \multicolumn{4}{|l|}{ Medications at discharge, $\mathrm{n}(\%)$} \\
\hline Aspirin & $3039(92.3)$ & $88(90.7)$ & 0.58 \\
\hline Clopidogrel & $2662(81.0)$ & 78 (7906) & 0.73 \\
\hline Statins & $2760(84.4)$ & $70(72.9)$ & 0.002 \\
\hline Beta-blockers & $2566(78.7)$ & $62(65.3)$ & 0.002 \\
\hline Angiotensin-converting enzyme inhibitors & $2030(62.9)$ & $66(68.8)$ & 0.24 \\
\hline Angiotensin receptor blockers & $270(8.6)$ & $4(4.3)$ & 0.15 \\
\hline Aldosterone receptor blockers & $153(4.9)$ & $17(18.3)$ & $<0.001$ \\
\hline Loop diuretics & $613(19.7)$ & $53(57.6)$ & $<0.001$ \\
\hline Digoxin & $17(0.5)$ & 0 & 0.48 \\
\hline Nitrates & $581(18.6)$ & $25(27.2)$ & 0.04 \\
\hline Amiodarone & $232(7.0)$ & $24(24.2)$ & $<0.001$ \\
\hline
\end{tabular}

\section{Propensity-score-matched population}

Separate analyses on propensity-score-matched populations confirmed these data: 1-year mortality in propensityscore-matched cohorts of early survivors was significantly higher in those with CS at the acute stage (HR: 2.49, 95\% CI: $1.43,4.33, P=0.001$ ) (Figure 2 and Additional file 2 show this in more detail).
In contrast, 5-year mortality in propensity-score-matched cohorts of 1-year survivors was similar in patients with CS at the acute stage (25\%) and in those without (23\%) (HR: 1.11, 95\% CI: 0.65, 1.91, $P=0.69$ ) (Figure 3 and Additional file 3 show this in more detail). In this population, similar characteristics were correlated with long-term survival in patients with or without CS (see Additional file 4).

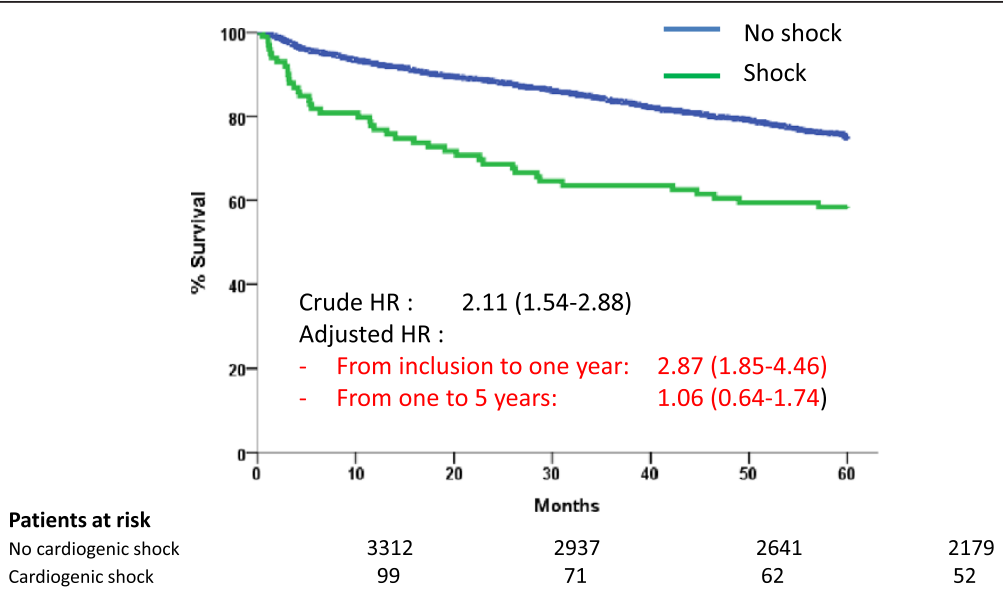

Figure 1 Five-year survival in patients surviving at 30 days and hospital discharge according to the cardiogenic shock status. $H R$, hazard ratio. 


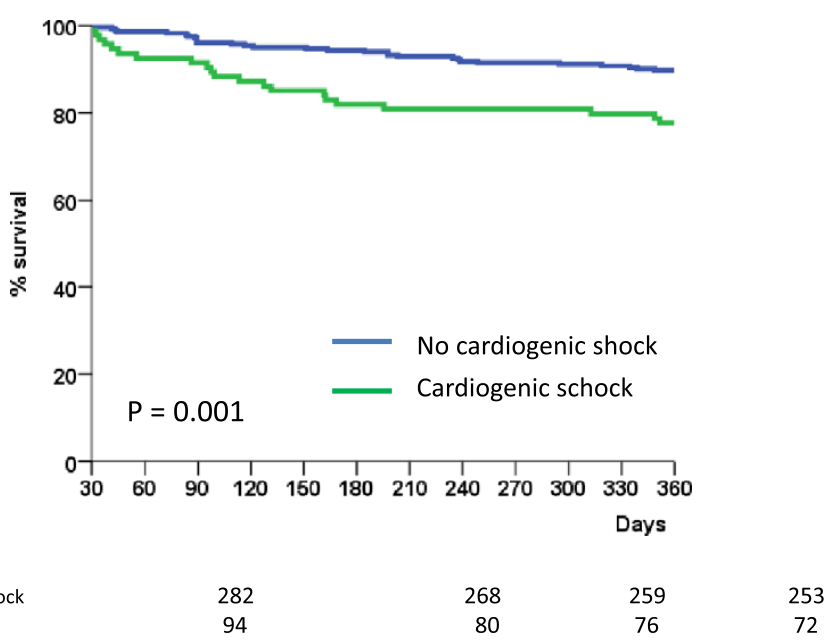

Figure 2 Landmark analysis: 1-year survival in propensity-score-matched cohorts of early survivors.

Predictors of 1-year death in early survivors with shock Using Cox multivariate analysis in the population of patients with CS, most prognostic factors for death at 1 year were un-modifiable (older age, diabetes mellitus, history of chronic kidney disease); likewise, patients with STEMI had a markedly increased risk, compared with NSTEMI patients (HR: 4.31; 95\% CI 1.38, 13.44). When STEMI patients were categorised according to use of reperfusion therapy at the acute stage, and compared with NSTEMI patients, lack of reperfusion in STEMI patients was related to poorer survival (HR: 6.44, 95\% CI: 1.91, 21.7 versus NSTEMI), while only a trend persisted for STEMI patients with reperfusion therapy (HR: 2.87, 95\% CI: 0.69, 11.9 versus NSTEMI). None of the discharge medications were associated with improved survival, although non-significant trends were observed for statins (HR: 0.43, 95\% CI: 0.10, 1.75) and clopidogrel (HR: 0.37, 95\% CI: 0.08, 1.60).

Predictors of 5-year death in 1-year survivors with shock In 1-year survivors who had CS at the acute stage, both older age, and previous history of coronary artery disease were associated with lower survival rate. Revascularisation procedures during the initial hospital stay were
Patients atrisk No cardiogenic shock Cardiogenicshock

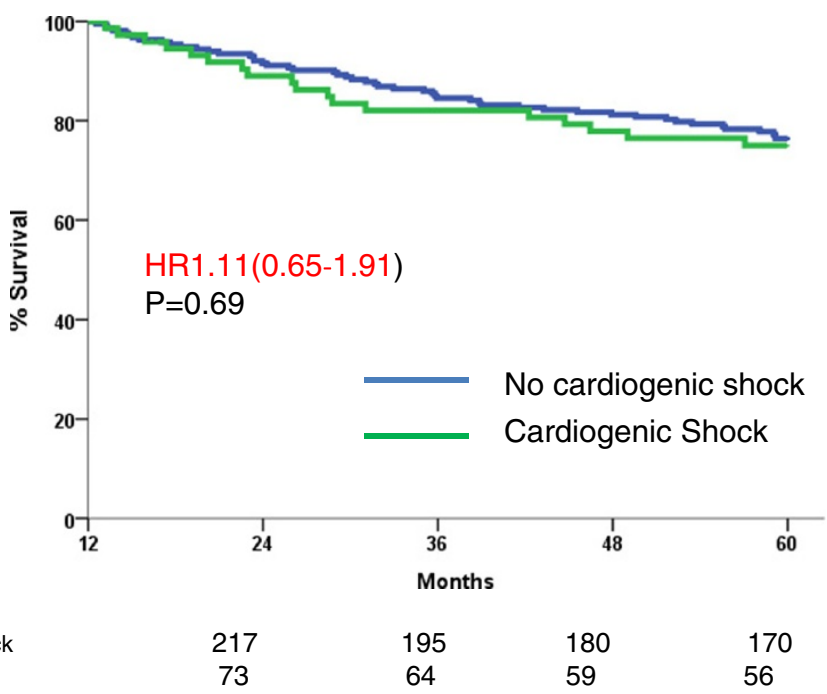

153 49

Figure 3 Landmark analysis: from one year to five years. Five-year survival in propensity-score-matched cohorts of patients surviving at one year. HR, hazard ratio. 
associated with lower 5-year mortality (HR: 0.12, 95\% CI: 0.03, 0.42).

\section{Discussion}

The main finding of the present study in a contemporary real-world population of AMI patients, is that early survivors of CS still have higher mortality at 1 year, compared with early survivors without CS. Beyond 1 year, however, mortality up to 5 years becomes comparable between patients with or without CS at the acute stage, although the former have a more severe clinical profile. Mortality is higher in STEMI patients with CS than in NSTEMI patients with CS, and early revascularization is associated with better long-term survival.

Most data available on the long-term outcome of CS patients surviving the early phase of AMI were obtained before major changes in AMI treatment were widely implemented [3-5]. They are derived from populations selected for inclusion into randomized trials, or less frequently from real-life populations. Patient inclusion was usually before the year 2000, at a time when outcomes were notably poorer than nowadays [12].

Despite improved early management, early mortality in CS patients remains considerably higher than that of patients without CS $[2,13]$. In prior studies the reported overall long-term survival of CS patients surviving the early period varies widely from $12 \%$ to $73 \%$ at 5 years $[4-6,14,15]$. In the large GUSTO-1 population of STEMI patients [5], mortality at 11 years in early survivors was $45 \%$ in patients with CS, compared with $31 \%$ in patients without CS. Patients included in the trial were relatively young and had to fulfill the trial inclusion criteria, which included early presentation after symptom onset, and all received intravenous fibrinolytic therapy. Our patients were enrolled in 2005, and more than $80 \%$ of patients underwent myocardial revascularization. This clearly differentiates our study, which is therefore clinically relevant in the current era.

To the best of our knowledge, there has been only one recent report on a large contemporary series of patients with long-term follow up after CS [16]. The data, gathered from the CRUSADE registry cross-linked with the Medicare administrative database, were limited to older ( $\geq 65$ years) patients and only included NSTEMI patients. Four-year survival in CS early survivors was $48 \%$, compared with $56.5 \%$ in non-CS survivors. Another novelty of our study is that both STEMI and NSTEMI patients were included.

In this population, 5-year survival of CS patients alive after the acute phase was $59 \%$. Improvements in primary angioplasty and adjunctive pharmacotherapy are likely to explain higher survival rates $[4,5]$ in recent series. Of note, a majority of our patients had undergone myocardial revascularization during the initial hospital stay and early revascularization was associated with better longterm survival. In the SHOCK trial, early revascularization significantly reduced 6-year mortality in early survivors by 41\% (absolute risk reduction: 18\%) [4]. Our study underlines the potential interest of performing immediate coronary reperfusion in CS, as it is also associated with improved long-term survival. In this regard, developing networks for the management of AMI patients, particularly for the most severely affected patients, seems important to improve both early and long-term outcomes.

A consistent finding in all studies, including ours, is similar mortality beyond 1 year in patients with or without CS, regardless of the period studied or the inclusion of STEMI or NSTEMI patients. The GUSTO-1 study confirmed similar long-term survival between CS and non-CS patients who were alive at 1 year. In the much more recent CRUSADE registry in NSTEMI patients 65 years of age or older, no difference was observed between patients with or without CS, once they had survived the first months following the acute episode [16]. The reasons for late survival becoming similar are unclear, especially when considering the difference in LVEF between patients who have developed CS and those who have not. Mechanisms leading to CS include left ventricular dysfunction, systemic inflammatory response, activation of complement, release of cytokines, and expression of inducible nitric oxide synthase $[4,14,17]$. The recent IABP SHOCK II trial confirmed the presence of a high degree of inflammatory response in CS patients, irrespective of the management strategy, and that CS could be present even in the absence of profound LV dysfunction (median LVEF 35\%) [17]. The resolution of severe ischaemia and/or neurohormonal abnormalities may explain the potential reversibility of shock $[4,14]$. Also, the increasing use of medications such as betablockers, ACE-inhibitors, ARBs or aldosterone blockers in patients with CS during the hospital admission may have participated in their improved long-term survival; of note, the percentage of patients with CS receiving beta-blockers increased from $68 \%$ at discharge to $81 \%$ at one year, and the percentage of patients receiving either ACE-inhibitors or ARBs marginally increased from $78 \%$ to $81 \%$.

\section{Strengths and limitations}

Our study has the usual limitation of observational data. Namely, no causality can be inferred from the associations we observed. In addition, the sample size of patients with CS was small, and subgroup analyses were therefore unrealistic. We excluded patients admitted more than $48 \mathrm{~h}$ after symptoms onset. These patients could have developed CS. Likewise, we were not able to assess the potential effect of implantable cardioverter defibrillators or of cardiac resynchronization therapy, as very few patients were implanted during the initial hospital stay (one in the 
CS group and six in the patients without CS). Conversely, our population was extremely well-characterized and reflected a real-world population. Also, it included both STEMI and NSTEMI patients and the rate of patients lost to follow up was low.

\section{Conclusion}

The long-term outcome for early survivors of CS is worse than that of patients without CS. Patients who survive the first year after the acute event, however, have a 5-year survival rate similar to that of non-CS patients. This divergence between early, semi-early, and long-term outcomes has remained consistent since the late 1980s and underlines the importance of improving short-term outcome by the early detection and management of CS, including early myocardial revascularisation.

\section{Key messages}

- Early survivors of CS still have a higher mortality at 1 year, compared with early survivors without CS

- Beyond 1 year, however, mortality up to 5 years becomes comparable between patients with or without CS at the acute stage, although the former have a more severe initial profile

- Mortality is higher in STEMI patients with CS than in NSTEMI patients with CS

- Early revascularization is associated with better long-term survival in CS patients following acute myocardial infarction

\section{Additional files}

\section{Additional file 1: Baseline, in-hospital and 1-year characteristics of the patients alive at one year.}

Additional file 2: Propensity-score-matched cohorts of patients alive at hospital discharge and 30 days.

Additional file 3: Propensity-score-matched cohorts of patients alive at one year.

Additional file 4: Characteristics of patients in the propensityscore-matched cohorts of 1-year survivors according to survival status at 5 years.

\section{Abbreviations}

ACE: angiotensin-converting enzyme; AMI: acute myocardial infarction; ARB: angiotensin-receptor blocker; AV: atrio-ventricular; CS: cardiogenic shock; FAST-MI: French registry of Acute ST-elevation and non-ST-elevation Myocardial Infarction; LVEF: left ventricular ejection fraction; MI: myocardial infarction; NSTEMI: non-segment-elevation myocardial infarction; STEMI: segment-elevation myocardial infarction.

\section{Competing interests}

N Aissaoui, none; E Puymirat, none; T Simon, research grants: Astra-Zeneca, Daiichi-Sankyo, Eli-Lilly, Glaxo-Smith-Kline, MSD, Novartis, Pfizer, sanofi-aventis, and Servier and speaker and consulting fees: AstraZeneca, Bayer-Schering, Eli-Lilly, and Sanofi-Aventis; E Bonnefoy, significant research grants: Astra-Zeneca, Daiichi-Sankyo, Gambro; D Angoulvant, research grants: Eli-Lilly, Abbott and speaker and consulting fees: Astra Zeneca, Eli-Lilly, Novartis, Bayer, MSD, Amgen, Pfizer; F Schiele, none; H Benamer, none; P Quandalle, none; F Prunier, Servier, significant research grant; E Durand, none; L Berard, none; D Blanchard, none; N Danchin, Servier, significant, research grant; Pfizer, significant, research grant; Sanofi-Aventis, significant, research grant.

\section{Authors' contributions}

NA conceived the study, drafted the manuscript, wrote the manuscript and revised the manuscript. EP helped to draft the manuscript and perform statistical analysis and helped to revise the manuscript. TS helped in acquisition of data, drafting the manuscript and statistical analysis. EBC helped in acquisition of data, and helped to conceive the study and correct the manuscript. DA helped in acquisition of data, interpretation of data and correction of the manuscript. FS participated in the design of the study, performed the statistical analysis and helped to revise the manuscript. $\mathrm{HB}$ helped in acquisition of data, interpretation of data, and drafting and revising the manuscript. PQ helped in acquisition of data, drafting the manuscript and performing the revision. FP helped in acquisition of data, drafting the manuscript and statistical analysis. ED helped in acquisition of data and revised it critically for important intellectual content. LB helped in acquisition of data, drafting the manuscript and statistical analysis. DB helped in acquisition and interpretation of data and revised it critically for important intellectual content. ND conceived of the study, performed the statistical analysis and the interpretation of data, and corrected the manuscript. All authors read the manuscript and gave final approval of the version to be published. All authors agree to be accountable for all aspects of the work in ensuring that questions related to the accuracy or integrity of any part of the work are appropriately investigated and resolved.

\section{Acknowledgements}

We are indebted to all patients having accepted to participate in the surveys, and to all participating physicians. The FAST-MI 2005 registry is a registry of the French Society of Cardiology, funded by unrestricted grants from Pfizer and Servier, and an additional grant from the Caisse Nationale d'Assurance Maladie. Special thanks to ICTA contract research organisation (Fontaine-lès-Dijon, France), and the devoted personnel of the URCEST (Assistance Publique des Hôpitaux de Paris and University Paris 6) and INSERM U 558 (Toulouse). Special thanks to Vincent Bataille, for his careful data management, to Benoît Pace (Société Française de Cardiologie) for his invaluable assistance in designing the electronic CRF, and to Geneviève Mulak (Société Française de Cardiologie) and Elodie Drouet, who supervised the patients' follow-up.

\section{Author details}

${ }^{1}$ Department of Cardiology, Assistance Publique-Hôpitaux de Paris, Hôpital Européen Georges Pompidou, Paris, France. ' Faculty of Medicine, University Paris Descartes, Paris, France. ${ }^{3}$ INSERM U970, Paris Cardiovascular Research Center PARCC, Paris, France. ${ }^{4}$ Assistance Publique-Hôpitaux de Paris,CHU St Antoine, Paris, France. ${ }^{5}$ INSERM U-698, Paris, France. ${ }^{6}$ UPMC, Paris 06, Paris, France. ${ }^{7}$ Intensive Cardiac Care Unit Hôpital Cardio-pneumologique, Lyon, France. ${ }^{8}$ Department of Cardiology CHRU de Tours and EA 4245 Faculty of Medicine, University François Rabelais, Tours, France. ${ }^{9} \mathrm{CHU}$ Besancon, Besancon, France. ${ }^{10}$ Hôpital Privé Jacques Cartier, Massy, France. ${ }^{11}$ Hôpital Victor Provo, Roubaix, France. ${ }^{12}$ Department of Cardiology, CHU Angers, and I'UNAM Université, Laboratoire Cardioprotection Remodelage et Thrombose, Angers, France. ${ }^{13} \mathrm{CHU}$ de Rouen, Rouen, France. ${ }^{14} \mathrm{Clinique}$ St Gatien, Tours, France.

Received: 25 April 2014 Accepted: 28 August 2014

Published online: 19 September 2014

\section{References}

1. Babaev A, Frederick PD, Pasta DJ, Every N, Sichrovsky T, Hochman JS, for the NRMI Investigators: Trends in management and outcomes of patients with acute myocardial infarction complicated by cardiogenic shock. JAMA 2005, 294:448-454.

2. Aissaoui N, Puymirat E, Tabone $X$, Charbonnier B, Schiele F, Lefèvre T, Durand E, Blanchard D, Simon T, Cambou JP, Danchin N: Improved outcome of cardiogenic shock at the acute stage of myocardial infarction: a report from the USIK 1995, USIC 2000, and FAST-MI French nationwide registries. Eur Heart J 2012, 33:2535-2543.

3. Goldberg RJ, Spencer FA, Gore JM, Lessard D, Yarzebski J: Thirty-year trends (1975 to 2005) in the magnitude of, management of, and hospital death rates associated with cardiogenic shock in patients with acute 
myocardial infarction: a population-based perspective. Circulation 2009, 119:1211-1219.

4. Hochman JS, Sleeper LA, Webb JG, Dzavik V, Buller CE, Aylward P, Col J, White HD, SHOCK Investigators: Early revascularization and long-term survival in cardiogenic shock complicating acute myocardial infarction. JAMA 2006, 295:2511-2515.

5. Singh M, White J, Hasdai D, Hodgson PK, Berger PB, Topol EJ, Califf RM, Holmes DR Jr: Long-term outcome and its predictors among patients with ST-segment elevation myocardial infarction complicated by shock: insights from the GUSTO-I trial. J Am Coll Cardiol 2007, 50:1752-1758.

6. Tedesco JV, Williams BA, Wright RS, Reeder GS, Dvorak D, Kopecky SL, Miller WL, Coronary Care Unit Group: Baseline comorbidities and treatment strategy in elderly patients are associated with outcome of cardiogenic shock in a community-based population. Am Heart J 2003, 146:472-478.

7. Cambou JP, Simon T, Mulak G, Bataille V, Danchin N: The French registry of Acute ST elevation or non-ST-elevation Myocardial Infarction (FAST-MI): study design and baseline characteristics. Arch Mal Coeur Vaiss 2007, 100:524-534.

8. Danchin N, Coste P, Ferrières J, Steg PG, Cottin Y, Blanchard D, Belle L, Ritz B, Kirkorian G, Angioi M, Sans P, Charbonnier B, Eltchaninoff H, Guéret $P$, Khalife K, Asseman P, Puel J, Goldstein P, Cambou JP, Simon T, FAST-Ml Investigators: Comparison of thrombolysis followed by broad use of percutaneous coronary intervention with primary percutaneous coronary intervention for ST-segment-elevation acute myocardial infarction: data from the french registry on acute ST-elevation myocardial infarction (FAST-MI). Circulation 2008, 118:268-276.

9. Antman EM, Anbe DT, Armstrong PW, Bates ER, Green LA, Hand M, Hochman JS, Krumholz HM, Kushner FG, Lamas GA, Mullany CJ, Ornato JP, Pearle DL, Sloan MA, Smith SC Jr, Alpert JS, Anderson JL, Faxon DP, Fuster V, Gibbons RJ, Gregoratos G, Halperin JL, Hiratzka LF, Hunt SA, Jacobs AK, for the American College of Cardiology/American Heart Association Task Force on Practice Guidelines (Writing Committee to Revise the 1999 Guidelines for the Management of Patients With Acute Myocardial Infarction: ACC/AHA guidelines for the management of patients with ST-elevation myocardial infarction: executive summary: a report of the American College of Cardiology/American Heart Association Task Force on Practice Guidelines. Circulation 2004, 110:588-636

10. Van de Werf F, Bax J, Betriu A, Blomstrom-Lundqvist C, Crea F, Falk V, Filippatos G, Fox K, Huber K, Kastrati A, Rosengren A, Steg PG, Tubaro M, Verheugt $F$, Weidinger $F$, Weis M, ESC Committee for Practice Guidelines (CPG), Vahanian A, Camm J, De Caterina R, Dean V, Dickstein K, Filippatos G, Funck-Brentano C, Hellemans I, Kristensen SD, McGregor K, Sechtem U, Silber S, Tendera M, et al: Management of acute myocardial infarction in patients presenting with persistent ST-segment elevation: the Task Force on the Management of ST-Segment Elevation Acute Myocardial Infarction of the European Society of Cardiology. Eur Heart J 2008, 29:909-945

11. Dafni U: Landmark analysis at the 25-year landmark point. Circ Cardiovasc Qual Outcomes 2011, 4:363-371.

12. Puymirat $E$, Simon $T$, Steg PG, Schiele F, Guéret $P$, Blanchard D, Khalife $K$, Goldstein P, Cattan S, Vaur L, Cambou JP, Ferrières J, Danchin N, USIK USIC 2000 Investigators; FAST MI Investigators: Association of changes in clinical characteristics and management with improvement in survival among patients with ST-elevation myocardial infarction. JAMA 2012, 308:998-1006

13. Jeger RV, Radovanovic D, Hunziker PR, Pfisterer ME, Stauffer JC, Erne P, Urban P, AMIS Plus Registry Investigators: Ten-year trends in the incidence and treatment of cardiogenic shock. Ann Intern Med 2008, 149:618-626.

14. Hochman JS: Cardiogenic shock complicating acute myocardial infarction: expanding the paradigm. Circulation 2003, 107:2998-3002.

15. Lindholm MG, Køber L, Boesgaard S, Torp-Pedersen C, Aldershvile J, Trandolapril Cardiac Evaluation study group: Cardiogenic shock complicating acute myocardial infarction; prognostic impact of early and late shock development. Eur Heart J 2003, 24:258-265.
16. Bagai A, Chen AY, Wang TY, Alexander KP, Thomas L, Ohman EM, Hochman JS, Peterson ED, Roe MT: Long-term outcomes among older patients with non-ST-segment elevation myocardial infarction complicated by cardiogenic shock. Am Heart J 2013, 166:298-305.

17. Thiele H, Zeymer U, Neumann FJ, Ferenc M, Olbrich HG, Hausleiter J, Richardt G, Hennersdorf M, Empen K, Fuernau G, Desch S, Eitel I, Hambrecht R, Fuhrmann J, Böhm M, Ebelt H, Schneider S, Schuler G, Werdan K, IABP-SHOCK II Trial Investigators: Intraaortic balloon support for myocardial infarction with cardiogenic shock. N Engl J Med 2012, 367:1287-1296.

doi:10.1186/s13054-014-0516-y

Cite this article as: Aissaoui et al:: Long-term outcome in early survivors of cardiogenic shock at the acute stage of myocardial infarction: a landmark analysis from the French registry of Acute ST-elevation and non-ST-elevation Myocardial Infarction (FAST-MI) Registry. Critical Care 2014 18:516.

\section{Submit your next manuscript to BioMed Central and take full advantage of:}

- Convenient online submission

- Thorough peer review

- No space constraints or color figure charges

- Immediate publication on acceptance

- Inclusion in PubMed, CAS, Scopus and Google Scholar

- Research which is freely available for redistribution

Submit your manuscript at www.biomedcentral.com/submit
C BioMed Central 